\title{
Leucocyte Count: A Window to Periodontal Disease? A Pilot Study
}

\author{
Dr. soumya. K. Nair, Dr. supriya Ebenezer, Dr. MD jalaluddin, Dr. Mohamed \\ Faizuddin, Dr. Jayanthi. D
}

\section{Introduction}

Periodontal disease is defined as an inflammatory reaction to a microbial infection associated with dental plaque that, results in tissue loss. Neutrophils or polymorphonuclear leucocytes (PMNs) play a critical role as a part of the innate immune response acting as a first line of defense against these invading microbes[1]. The presence of leucocytes in the oral cavity has attracted interest for many years. Calonius in 1958 compared the salivary leucocyte count in patients with healthy and inflamed gingiva and edentulous patients and found that the levels were least in edentulous patients and highest in patients with gingivitis thus suggesting that leucocytes enter the saliva through the gingival sulcus[2]. This was also confirmed by studies done by Schiott and Loe in 1970[3]. In the healthy periodontium of both humans and experimental animals, PMNs have been demonstrated migrating towards or residing within the sulcular and junctional epithelium and within the underlying connective tissue[4]. With plaque accumulation and the development of clinical inflammation there is an increase in the number of leucocytes present in the lesion[5,6,7]. The location of PMNs at the plaque interface, their phagocytic activity and signs of lysosomal enzyme release give morphological evidence that these cells, may on one hand, protect the tissue from bacterial attack but on the other hand, may induce tissue damage and increased inflammation via release of lysosomal enzymes. Thus, high numbers of subgingival leucocytes could possibly indicate an active periodontal lesion[8]. Subgingival leucocyte counts maybe useful in identifying sites with active periodontal disease.Many efforts have been made to find reliable parameters for the diagnosis and prognosis in periodontology. Among these the biochemical parameters especially the enzymatic concentrations in the gingival crevicular fluid (GCF) pose as a potential tool to assess disease activity. Interestingly the most common enzymes found in the GCF are released from the poly morhonuclear leucocytes $(\mathrm{PMN})$ and these cells play a pivotal role in the pathogenesis of periodontitis[9] and their numbers vary from patient to patient as well as from site to site.

A pilot study is defined as a smaller version of a larger study that is conducted to prepare for that study. A pilot study can involve pretesting a research tool, like a new data collection method. It can also be used to test an idea or hypothesis. Pilot studies are used as feasibility studies, to ensure that the ideas or methods behind a research idea are sound, as well as to "work out the kinks" in a study protocol before launching a larger study. They are also used in clinical trials to see if the study can be carried out on a larger scale.The present pilot study is undertaken to estimate the number of PMNs in the GCF of periodontitis patients and compare it with healthy controls so as to know whether the levels of PMN count can serve as diagnostic marker of disease activity.

\section{Materials And Methods}

This study was conducted in the Department of Periodontics and Oral Implantology,M R Ambedkar dental college and hospital,Bangalore, Karnataka, India. Twenty subjects (12 females and 8 males) in the age range 20 to 65 years were recruited for the study.Informed written consent was obtained from all subjects and ethical clearance was obtained from the ethical board of this institution. Two groups with 10 subjects each were designated as controls(clinically healthy) and cases(chronic generalized periodontitis) respectively, according to the Gingival Index (Loe andSillness, 1963) and Russell's Periodontal Index(1956). ${ }^{16,17}$

\section{Subject inclusion criteria:}

1. Subjects with periodontal disease. (chronic generalized periodontitis).

2. Subjects who were systemically healthy.

3. No invasive periodontal therapy in the past six months.

\section{Subject exclusion criteria:}

1. Systemic diseases like diabetes mellitus.

2. Pregnant subjects.

3. Smokers and alcoholics.

4. Presence of disease with possible effects on the immune system like chronic infection or cancer. 
5. Treatment with any drugs that might alter PMN number or function.

6. Use of any antibiotics during the study period or in the recent past and subjects who have undergone noninvasive periodontal therapy.

7. Subjects with recent history of fever or illness

8. Presence of carious lesion or any kind of mucosal ulceration.

A dental and medical history was compiled for all subjects with an oral examination. Clinical measurement of probing depth at four sites per tooth was performed by a single investigator who did all data collection and examinations.

Collection of GCF: The subjects were asked to gargle their mouth vigorously with water to cleanse the teeth of loosely adherent debris. Samples of GCF were obtained from any healthy site by placing color coded, calibrated, volumetric, micro capillary pipettes with $0.5 \mathrm{ml}$ range, obtained from Sigma chemical company,St louis, Missouri, U.S.A. The test site was dried and isolated with cotton rolls. Volumetric micropipettes were placed extra crevicularly at the entrance of the gingival crevice and for each subject, and the GCF sample collected. The pipettes which were contaminated with blood or saliva were discarded. Pooled volume of GCF was collected from healthy subjects and whereas for periodontitis site samples were collected from sites exhibiting severe inflammation and deepest probing depth $(>5 \mathrm{~mm})$. Test sites which did not express any volume of GCF and Micropipettes contaminated with blood and saliva were not included in the study. Once the GCF was collected it was immediately transferred onto a slide. A thin smear was prepared and stained using Leishmanns stain. It was then viewed under a light microscope with oil immersion lens (100x magnification). The number of neutrophils per hundred cells were counted in a field. The above procedure was done for ten healthy patients and ten chronic periodontitis patients.

\section{Statistical Analysis}

A student $t$ test was done to compare the values of the cases and controls and a statistical significance was established at $\mathrm{p}<0.5$

\section{Results}

Mean percentage of PMNs in the cases or periodontits patients $(74+2.4289)$ was significantly higher than those with healthy periodontium $(70.9+1.092)$ or controls at $\mathrm{p}<0.01$.However the percentage of lymphocytes was higher in healthy $(27.3+2.5882)$ compared to the periodontitis patients $(23.9+2.8442)$. $($ table 1 and graph 1)

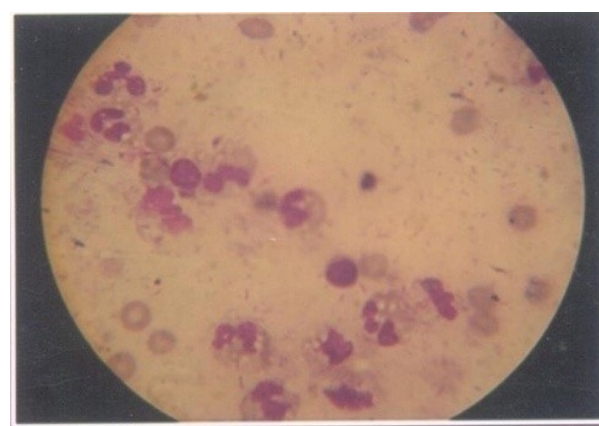

fig 1 Microscopic field showing leucocytes in periodontitis

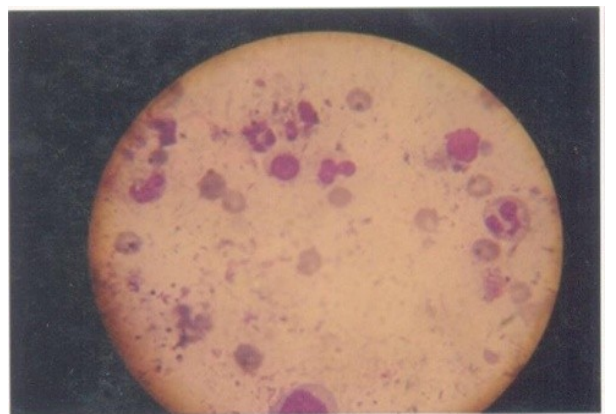

fig 2 Microscopic field showing leucocytes in healthy 


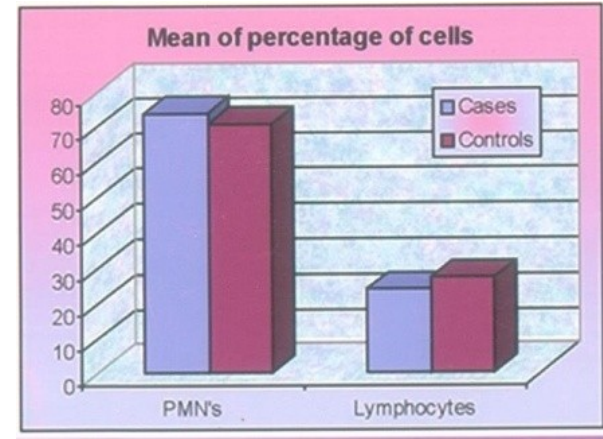

Graph 1. Mean percentage of cells in cases \& controls

\begin{tabular}{c|c|c|c|}
\hline & CASES & CONTROLS & PVALUE \\
\hline PMN(Mean \pm SD) & $74 \pm 2.4289$ & $70.9 \pm 1.9205$ & $<0.01$ \\
\hline $\begin{array}{c}\text { LYMPHOCYTES } \\
\text { (Mean+SD) }\end{array}$ & $23.9 \pm 2.8442$ & $27.3 \pm 2.2552$ & $<0.01$ \\
\hline
\end{tabular}

Tabel 1. Mean values of PMN and lymphocytes in cases and controls

\section{Discussion}

The main source of neutrophils in the oral cavity are from those migrating from the gingival sulcus[2]. The results of the present study showed increase in the PMN leucocytes in GCF of cases or chronic generalized periodontitis patients compared to healthy controls. This could be attributed to increase surface area of ulcerated epithelium and hence increase in the migration of PMN leucocytes though the ulcerated epithelium in the cases[10]. Using neutrophil counts in the GCF to evaluate the periodontal disease activity has been used in earlier studies and has shown a positive correlation with the probing pocketdepth. ${ }^{15(11)}$ There are a number of ways of collecting GCF for neutrophil estimation. The use of Styroflex strips, might not give accurate results, due to the clumping of the cells. ${ }^{4}$ The washing method suggested by Skapski and Lehner in 1976, and by Salonen and Paunio in 1991 has a shortcoming that the dilution factor cannot be determined accurately and thus not an ideal method. ${ }^{18,19(12,13)}$ In our study we have used colour coded capillary micropippetes to collect GCF samples since it is one of the novel methods of collecting GCF sample without the disadvantages of the above mentioned methods. There is lesser contamination with blood and saliva and also ours is an extra crevicular method of GCF collection. The main advantage of this is the collection of unstimulated GCF which can give us the near perfect picture of what is actually going on under the sulcular epithelium. Our study showed a greater presence of PMNs in the cases as compared to the controls in accordance with the study by Anderson \& Cimasoni in 1993. ${ }^{15(11)}$ But the Anderson study has correlated the PMN with the mild, moderate and severe periodontitis and shown a positive corerelation of the PMNs with deeper pocket depts. Also in our study we have taken only healthy and periodontitis into consideration since gingivitis is a reversible process which is more dependent on the oral hygiene and plaque control unlike periodontitis which is an irreversible process unless treated. Hence for a real picture of the actual ongoing disease process the collection of GCF from a periodontitis patient is any day better since periodontitis is the chronic inflammatory infectious disease that undergoes periods of activity and quiescence. The GCF from periodontitis patients only can give a clearer view of the underlying pathophysiology.

The present study also showed a reversal of the findings in case of the lymphocytes that were larger in number in the controls compared to the cases. In an animal study it was seen that repopulating lymphocytes are critical for modulating responses to superinfection. Once inflammation ensues in normal mice, lymphocytes disappear in response to DP1 receptor activation by prostaglandinD2 .However, upon resolution,lymphocytes repopulate the cavity and are dispensable for resolution, as inflammation in mice resolve uniformly.Hence it can be concluded that lymphocytes are there in healthy sites probably to modulate the responses to superinfecction and to keep the site heathy ${ }^{14}$. Also since the sample size is really small there is always the benefit of doubt which is the limitation of the present study. However it was a pilot study conducted with the intention of conducting a further trial with larger sample size for a more coherent picture. Despite the tremendous development in microbiologic and immunologic diagnostic markers, most of them failed to show any clinical applicability. Microbiologic markers are fraught with technical difficulties especially when it comes to anaerobic culturing for periodontopathogens and takes time to obtain the results. Collection of gingival biopsy samples for immunologic markers has its own limitations. ${ }^{15}$ On the contrary chair side microscopic examination for the quantitative estimation of PMN leucocytes is not plagued by these limitations. 
At present the most commonly used diagnostic too list periodontal probing but it's a one dimensional measurement of a three dimensional space. Also, an error of $1 \mathrm{~mm}$ will result in $50 \%$ error, with the biggest advantage being speed of execution and immediacy of interpretation as compared to other microbiologic or immunologic methods. Periodontal probing provides clinical information regarding pocket depth and configuration, but periodontal pockets go through periods of exacerbation and quiescence. Periods of quiescence are characterized by reduced inflammatory response and reduced amount of bone and little or no loss of bone and connective tissue attachment and the opposite, in periods of activity.Thus it is is important to know current disease activity, which will have an implication on treatment options. These considerations suggest that the advantage of probing though acceptable and irreplaceable in routine periodontal practice is deficient when disease activity is to be evaluated. Hence alternate measures to assess periodontal disease activity can be used based on indicators of inflammatory process ${ }^{15}$. GCF neutrophils count could be used to assess the disease activity as a simple chairside diagnostic tool .However the disadvantage of this method is that it is not a method with very high specificity. Also the results of a pilot study cannot be always right but it definitely throws light on the perspective of a future trial or research with a larger sample size.

Further studies could be directed to develop a chairside color changing agent similar to a disclosing agent that stains neutrophils in plaque which could help screen and monitor periodontitis subjects. Clinicians can use the plaque neutrophils to check the disease activity in subjects on supportive periodontal therapy. This could be further developed for screening of aggressive periodontitis subjects who have quantitative neutrophil abnormality.

\section{Conclusion}

This study demonstrates that it is possible to collect and quantify oral neutrophils by a single, rapid, noninvasive assay using capillary micropippetes. Neutrophils are found in higher numbers in GCF of chronic severe periodontitis disease, a finding that reflects the inflammatory nature of the disease process. However further trials with larger sample sizes are required for a more coherent picture. Hence this pilot study shows scope for future research in this area.

\section{References}

[1]. Dyke TE, Vaikuntum J. Neutrophil function anddysfunction in periodontal disease. CurrOpinPeriodontol1994; 28:19-27.

[2]. Calonius PEB. The leucocyte count in saliva. Oral Surg1958;11: 43-6.

[3]. Schiott CR, Loe H. The origin and variation of number ofleucocytes in human saliva. J Periodont Res 1970; 5: 36-41.

[4]. Attstrom R. Presence of leucocyte in crevices of healthyand chronically inflamed gingivae. J Periodont Res 1970;5:42-7.

[5]. Shroeder HE, Graf-de Beer M, Attstrom R. Initial gingivitisin dogs. J Periodont Res 1975;10:128-42.

[6]. Lindhe J, Rylander H. Experimental gingivitis in young dogs.Scan J Dent Res 1975; 83: 314-26.

[7]. Payne WA, Page RC, Ogilvie AC, Hall WB. Histologicalfeatures of initial and early stages of experimental gingivitisin man. J Periodont Res 1975; 10: 51-64.

[8]. Miller DR., Lamster IB, Chasens AI. Role ofpolymorphonuclear leucocytes in periodontal health anddisease. J ClinPeriodontol 1984; 11: 1-15.

[9]. Newman MG, Takei HH, Klokkevold PR, Carranza FA Jr.,editors. Carranza's Clinical Periodontology.10th edition, St.Louis. Missouri Saunders. Elsevier Inc; 2006:444-5.

[10]. Skougaard MR, Klinkhammer JM. Correlation betweengingivitis and Orogranulocyte migratory rate. J Dent Res1969; 48:716-18.

[11]. Andersen PE, Cimasoni G. A rapid and simple method forcounting crevicularpolymorphonuclear leucocytes. JClinPeriodontol 1993; 20: 651-55.

[12]. Skapski H, Lehner T. A crevicular washing method forinvestigating immune components of crevicular fluid in man.J Periodont Res 1976; 11: 19-24.

[13]. Salonen JI, Paunio KU. An intracrevicular washing methodfor collection of crevicular contents. Scan J Dent Res 1991;99: 406-12.

[14]. Bellingan, Desmond Fitzgerald, Muhammad M. Yaqoob and Derek W. Gilroy,Ravindra Rajakariar, Toby Lawrence, Jonas Bystrom, Mark Hilliard, Paul Colville-Nash, GeoffInflammation.Novel biphasic role for lymphocytes revealed during resolving.http://bloodjournal.hematologylibrary.org/content/111/8/4184.full.html

[15]. Aspey DJ, Kaciroti N, LoescheWJ. The diagnosis of periodontal disease in private practice. J Periodontol 2006;77: 1572-81. 